Article

\title{
Nonclassical Symmetries of a Power Law Harry Dym Equation
}

\author{
Daniel J. Arrigo * and Andrea N. Weaver \\ Department of Mathematics, University of Central Arkansas, Conway, AR 72035, USA; aweaver3@cub.uca.edu \\ * Correspondence: darrigo@uca.edu; Tel.: +1-501-450-5668
}

Received: 31 January 2018; Accepted: 4 April 2018; Published: 6 April 2018

\begin{abstract}
It is generally known that classical point and potential Lie symmetries of differential equations can be different. In a recent paper, we were able to show for a class of nonlinear diffusion equation that the nonclassical potential symmetries possess all nonclassical symmetries of the original equation. We question whether this is true for the power law Harry Dym equation. In this paper, we show that the nonclassical symmetries of the power law Harry Dym equation and an equivalent system still possess special separate symmetries. However, we will show that we can extend the nonclassical method so that all nonclassical symmetries of the original power law Harry Dym equation can be obtained through the equivalent system.
\end{abstract}

Keywords: classical symmetry; nonclassical symmetry; Harry Dym equation

\section{Introduction}

Symmetry analysis plays a fundamental role in the construction of exact solutions to nonlinear partial differential equations. Based on the original work of Lie [1] on continuous groups, symmetry analysis provides a unified explanation for the seemingly diverse and ad-hoc integration methods used to solve ordinary differential equations. At the present time, there is extensive literature on the subject, and we refer the reader to the books by Arrigo [2], Bluman and Kumei [3], Cherniha et al. [4], and Olver [5] .

A question that continues to arise is in the symmetries of a particular equation and an equivalent system. For example, the nonlinear diffusion equation

$$
u_{t}=\left(u^{m} u_{x}\right)_{x}
$$

and the equivalent system

$$
v_{t}=u^{m} u_{x}, \quad v_{x}=u
$$

possess different symmetries for different values of $m$. If $m=-4 / 3$ then (1) possesses extra symmetries (see [3,6,7]) whereas if $m=-2$, then the system (2) possesses extra symmetries ([8]). A further example is found with the wave equation

$$
u_{t t}=\left(u^{m} u_{x}\right)_{x}
$$

and the equivalent system

$$
v_{t}=u^{m} u_{x}, \quad v_{x}=u_{t}
$$

where the powers $m=-4 / 3$ and $m=-4$ show themselves as special ([9-11]). The idea that point symmetries can be gained or loss when one considers equivalent potential systems has been an active area of research (see, for example, [12-16] and references within) and has been applied to a number of equations including the nonlinear heat and wave equations $([8,17-19])$, the equations of planar gas dynamics $([13,20,21])$ and the equations of nonlinear elasticity [22] to name just a few. 
Arrigo et al. [23] asked whether this holds true for nonclassical symmetries; that the nonclassical symmetries of a particular equation and a system equivalent (termed nonclassical potential symmetries) are different. They were able to show that for a large class of nonlinear diffusion equation, the nonclassical potential symmetries contains the nonclassical symmetries of the original equation. They were also able to show this for a nonlinear wave equation and highlighted a special case where the opposite was true. In this paper, we continue our study where we consider a power law Harry Dym equation and an equivalent system.

\section{Classical Symmetries of the Harry Dym Equation}

The power law Harry Dym equation

$$
\left(u^{m}\right)_{t}=u_{x x x}
$$

admits the symmetry generator

$$
\Gamma=T \frac{\partial}{\partial t}+X \frac{\partial}{\partial x}+U \frac{\partial}{\partial u}
$$

where $T, X$ and $U$ are

$$
T=3 c_{1} t+c_{0}, \quad X=b_{1} x+b_{0}, \quad U=\frac{3\left(c_{1}-b_{1}\right) u}{m-1},
$$

for general powers $m(m \neq 1)$ and in the special case $m=-2$,

$$
T=3 c_{1} t+c_{0}, \quad X=b_{2} x^{2}+b_{1} x+b_{0}, \quad U=\left(2 b_{2} x+b_{1}-c_{1}\right) u,
$$

where $c_{i}, b_{i}$ are arbitrary constants (Bruzon et al. [24]) noting the additional generator in (8)

$$
\Gamma=x^{2} \frac{\partial}{\partial x}+2 x u \frac{\partial}{\partial u} .
$$

We note that the symmetries in (8) can be connected by a nonlocal transformation to the ones that appear in the work of Tychynin [25] for the Harry Dym type equation

$$
w_{t}=w_{x x}^{-3 / 2} w_{x x x} .
$$

The equivalent system

$$
v_{t}=u_{x x}, \quad v_{x}=u^{m}
$$

admits the symmetry generator

$$
\Gamma=T \frac{\partial}{\partial t}+X \frac{\partial}{\partial x}+U \frac{\partial}{\partial u}+V \frac{\partial}{\partial v}
$$

where $T, X, U$ and $V$ are given by

$$
T=3 c_{1} t+c_{0}, \quad X=b_{1} x+b_{0}, \quad U=\frac{3\left(c_{1}-b_{1}\right) u}{m-1}, \quad V=\frac{\left(3 c_{1} m-(2 m+1) b_{1}\right) v}{m-1}+c_{2}
$$

for $m \neq 1$ and in the special case of $m=-1 / 2$,

$$
T=3 c_{1} t+c_{0}, \quad X=b_{1} x+F(t, v), \quad U=2\left(b_{1}-c_{1}\right) u+2 \sqrt{u} F_{v}(t, v), \quad V=c_{1} v+c_{2},
$$

where again $c_{i}, b_{i}$ are arbitrary constants and $F(t, v)$ satisfies $F_{t}+2 F_{v v v}=0$ (Gardarais and Bruzon [26]). 
It is interesting to note that the appearance of the arbitrary function $F(t, v)$ in the symmetry (13) satisfies a linear PDE. This indicates that in the case $m=-1 / 2$, the original system (10) is linearizable (Bluman and Kumei [27]). In fact, under the point transformation

$$
t=\bar{t}, \quad x=\bar{v}, \quad u=\bar{u}, \quad v=\bar{x},
$$

where $\bar{u}=\bar{u}(\bar{t}, \bar{x})$ and $\bar{v}=\bar{v}(\bar{t}, \bar{x}),(10)$ is transformed to

$$
\bar{v}_{\bar{t}}=-\frac{\bar{u}_{\bar{x} \bar{x}}}{\bar{v}_{\bar{x}}}+\frac{\bar{u}_{\bar{x}} \bar{v}_{\bar{x} \bar{x}}}{\bar{v}_{\bar{x}}^{2}}, \quad \bar{v}_{\bar{x}}=\bar{u}^{1 / 2},
$$

and elimination of $\bar{u}$ in (15) gives $\bar{v}_{\bar{t}}+2 \bar{v}_{\bar{x}} \bar{x} \bar{x}=0$.

As this example illustrates, there continue to be cases where the classical symmetries of a PDE and an equivalent system are different. We ask whether these differences persist when we extend the analysis to nonclassical symmetries.

\section{Nonclassical Symmetries of the Power Law Harry Dym Equation}

To calculate the nonclassical symmetries, we append to the original PDE (or system) an invariant surface condition(s) and seek invariance of both together [28]. For the single equation, we seek invariance of (5) and

$$
T u_{t}+X u_{x}=U
$$

whereas for the system, we seek invariance of (10) augmented with the pair of surface conditions

$$
T u_{t}+X u_{x}=U, \quad T v_{t}+X v_{x}=V .
$$

If $T \neq 0$, we can set $T=1$ without loss of generality which we do so here. The nonclassical method give rises to a set of determining equations for the functions $X$ and $U$ (or $X, U$ and $V$ for the system) which we need to solve. As we are dealing with two equations, the single Harry Dym Equation (5) and an equivalent system (10), we will be considered them separately.

The determining equations for the nonclassical symmetries of (5) are:

$$
\begin{aligned}
X_{u} & =0, \\
U_{u u} & =0, \\
U_{x u}-X_{x x} & =0, \\
m\left(U_{t}+3 U X_{x}\right) u^{m-1}+m(m-1) U^{2} u^{m-2}-U_{x x x} & =0, \\
m\left(X_{t}+3 X X_{x}\right) u^{m-1}+m(m-1) X U u^{m-2}+3 U_{x x u}-X_{x x x} & =0 .
\end{aligned}
$$

These are solved giving the following. For general powers $m$

$$
X=a(t) x+b(t), \quad U=c(t) u,
$$

where $a(t), b(t)$ and $c(t)$ satisfy

$$
\dot{a}+3 a^{2}+(m-1) a c=0, \quad \dot{b}+3 a b+(m-1) b c=0, \quad \dot{c}+3 a c+(m-1) c^{2}=0 .
$$

These are easily solved and are equivalent to the classical symmetries presented in (7). We also have two special cases: $m=-2$ and $m=2$. When $m=-2$, then

$$
X=a(t) x^{2}+b(t) x+c(t), \quad U=(2 a(t) x+d(t)) u,
$$

where $a(t), b(t), c(t)$, and $d(t)$ satisfy 


$$
\begin{gathered}
\dot{a}+3 a b-3 a d=0, \quad \dot{b}+3 b^{2}-3 b d=0, \\
\dot{c}+3 b c-3 c d=0, \quad \dot{d}+3 b d-3 d^{2}=0 .
\end{gathered}
$$

These are easily solved and are equivalent to the classical symmetries presented in (8). When $m=2$ then

$$
X=0, \quad U=Q(x),
$$

where $Q^{\prime \prime \prime}=2 Q^{2}$. This, to the best of our knowledge is a new result.

The determining equations for the nonclassical symmetries of (10) are:

$$
\begin{aligned}
V_{u}-u^{m} X_{u} & =0, \\
V_{x}+\left(V_{v}-X_{x}\right) u^{m}-X_{v} u^{2 m}-m U u^{m-1} & =0, \\
X_{u u} & =0, \\
U_{u u}-2 X_{x u}-2 X_{u v} u^{m}-m X_{v} u^{m-1} & =0, \\
X_{x x}+3 V X_{u}-2 X_{x u}-X V_{u}+X_{v v} u^{2 m}+\left(X_{x v}-2 U_{u v}-2 X X_{u}\right) u^{m}-m U_{v} u^{m-1} & =0, \\
V_{t}+2 V X_{x}-V U_{u}+U V_{u}+V V_{v}-U_{x x}-\left(X X_{v}+U_{v v}\right) u^{2 m} & - \\
\left(X_{t}+2 X X_{x}+U X_{u}-X U_{u}-V X_{v}+X V_{v}+2 U_{x v}\right) u^{m} & =0 .
\end{aligned}
$$

These are solved giving rise to the following results. For general powers $m(m \neq 1)$

$$
X=a(t) x+b(t), \quad U=\frac{(c(t)-a(t)) u}{m-1}, \quad V=c(t) v+d(t),
$$

where $a(t), b(t), c(t)$, and $d(t)$ satisfy

$$
\begin{aligned}
m \dot{a}+(2 m+1) a^{2}+(m-1) a c & =0, \\
m \dot{b}+(2 m+1) a b+(m-1) b c & =0, \\
m \dot{c}+(2 m+1) a c+(m-1) c^{2} & =0 \\
m \dot{d}+(2 m+1) a d+(m-1) c d & =0
\end{aligned}
$$

and are easily solved leading to a recovery of classical symmetries presented in (12). In the special case where $m=-1 / 2$, then

$$
X=\left(G_{v}+p(t)\right) x+H(t, v), \quad U=2 p(t) u+2 G_{v} \sqrt{u}, \quad V=G(t, v),
$$

where $G(t, v), H(t, v)$ and $p(t)$ satisfy

$$
\begin{array}{r}
G_{t}+3 G G_{v}-4 G_{v v v}=0 \\
G_{t v}+3 G_{v}^{2}+2 G_{v v v v}+3 p(t) G_{v}+\dot{p}=0 \\
H_{t}+3 H G_{v}+2 H_{v v v}=0 .
\end{array}
$$

This overdetermined system can be solved and are equivalent to the classical symmetries presented in (13).

As this example illustrates, the nonclassical symmetries continue to separate into cases where each (the single equation vs an equivalent system) posses special symmetries. Unlike the case for the nonlinear diffusion equation where the nonclassical symmetries of an equivalent system captures the nonclassical symmetries of the original PDE, it is simply not true for the Harry Dym equation. A natural question to ask is can we extend the nonclassical method so that the nonclassical symmetries of the equivalent system encompasses the nonclassical symmetries of the single equation? For example, the Harry Dym equation 


$$
\left(u^{-2}\right)_{t}=u_{x x x}
$$

admits the conditional symmetry operator

$$
\Gamma=\frac{\partial}{\partial t}+x^{2} \frac{\partial}{\partial x}+2 x u \frac{\partial}{\partial u} .
$$

The associated invariant surface condition is

$$
u_{t}+x^{2} u_{x}=2 x u
$$

It is an easy matter to show that (28) and (30) are compatible. In terms of an equivalent system

$$
v_{t}=u_{x x}, \quad v_{x}=u^{-2},
$$

we would append the invariant surface conditions

$$
u_{t}+x^{2} u_{x}=2 x u, \quad v_{t}+x^{2} v_{x}=V(t, x, u, v),
$$

for some $V$. However, there is no $V(t, x, u, v)$ such that (31) and (32) are compatible. However, if we change the second equation in (32) and consider

$$
u_{t}+x^{2} u_{x}=2 x u, \quad v_{t}-2 \frac{u_{x}}{x}=-\frac{2 u}{x^{2}}-\frac{x^{2}}{u^{2}}
$$

then (31) and (33) are in fact compatible. Thus, we propose that it is possible to extended the nonclassical method so that all the nonclassical symmetries of the original PDE (1) can be obtained through the equivalent system (2). We prove this in the next section.

\section{Nonclassical Method Extension}

In this section, we will show that it is possible to extend the nonclasical method as to obtain the nonclassical symmetries of the original single PDE (5). In fact, we will show this for the general class of PDEs

$$
(F(u))_{t}=u_{x x x} .
$$

The determining equations for the nonclassical symmetries of (34) are

$$
\begin{aligned}
U_{u u} & =0, \\
U_{x u}-X_{x x} & =0, \\
U^{2} F_{u u}+\left(U+t+3 U X_{x}\right) F_{u}-U_{x x x} & =0, \\
X U F_{u u}+\left(X_{t}+3 X X_{x}\right) F_{u}+3 U_{x x u}-X_{x x x} & =0,
\end{aligned}
$$

where $X=X(t, x)$ and $U=U(t, x, u)$. The equivalent system to (34) is

$$
v_{t}=u_{x x}, \quad v_{x}=F(u) .
$$

If we were to append to (36)

$$
u_{t}+X u_{x}=U, \quad v_{t}+X v_{x}=V,
$$

where $U=U(t, x, u)$ and $V=V(t, x, u, v)$ and require compatibility, we would obtain the determining equations for the nonclassical symmetries of the system. However, as shown in the previous section, we cannot obtain all the nonclassical symmetries of the original PDE. This was explicitly shown when 
$F=u^{m}$. In order to extend the nonclassical method, we now seek compatibility of (36) with the following first order system

$$
u_{t}+X u_{x}=U, \quad v_{t}=V\left(t, x, u, v, u_{x}\right)
$$

We have omitted the inclusion of $v_{x}$ in $V$ due to (36). On solving (36) and (38) for $v_{t}, v_{x}, u_{t}$ and $u_{x x}$, we obtain

$$
\begin{aligned}
v_{t} & =V\left(t, x, u, v, u_{x}\right), & v_{x} & =F(u), \\
u_{t} & =U-X u_{x}, & u_{x x} & =V\left(t, x, u, v, u_{x}\right) .
\end{aligned}
$$

Requiring compatibility of (39) (i.e., $\left(v_{t}\right)_{x}=\left(v_{x}\right)_{t}$ and $\left.\left(u_{t x}\right)_{x}=\left(u_{x x}\right)_{t}\right)$ gives rise to

$$
\begin{array}{r}
V_{x}+p V_{u}+F(u) V_{v}+V V_{p}+(p X-U) F^{\prime}(u)=0, \\
V_{t}+X V_{x}+U V_{u}+(V+X F) V_{v}+\left(U_{x}+p U_{u}-p X_{x}\right) V_{p}+ \\
\left(2 X_{x}-U_{u}\right) V-U_{x x}+p\left(X_{x x}-2 U_{x u}\right) p-U_{u u} p^{2}=0 .
\end{array}
$$

We now show that $V$ exists satisfying (40a) and (40b) if $X$ and $U$ satisfy (35). It suffices to show that (40a) and (40b) are compatibile. Solving (40a) and (40b) for $V_{t}$ and $V_{x}$ and imposing compatibility gives

$$
\begin{gathered}
3\left(U_{u u} p+U_{x u}-X_{x x}\right) V+U_{u u u} p^{3}+3 U_{x u u} p^{2} \\
+\left(X U F_{u u}+\left(X_{t}+3 X X_{x}\right) F_{u}+3 U_{x x u}-X_{x x x}\right) p \\
-\left(U^{2} F_{u u}+\left(U+t+3 U X_{x}\right) F_{u}-U_{x x x}\right)=0,
\end{gathered}
$$

which, by the virtue of (35) is identically satisfied showing that $V$ exists thus proving our claim.

\section{Conclusions}

It is well known that classical Lie symmetries for different equivalent equations can be different. In a previous paper, we questioned whether this is true if we extend the symmetries to include nonclassical symmetries. Although we were able to show for certain classes of equation, the nonclassical symmetries of an equivalent system captures the nonclassical symmetries of the single equation, in general this is not true. In this paper, we have considered the nonclassical symmetries of a power law Harry Dym equation and an equivalent system. We have shown that there continue to be special cases where special nonclassical symmetries exists for each (a single equation vs a equivalent system). However, we have shown in the case of a generalized Harry Dym equation, we can extend the nonclassical method to encompass the nonclassical symmetries of both the single equation and an equivalent system.

Acknowledgments: The second author gratefully acknowledges the support of an Arkansas SURF grant.

Author Contributions: The authors contributed equally to this work.

Conflicts of Interest: The authors declare no conflict of interest.

\section{References}

1. Lie, S. Klassifikation und Integration von gewohnlichen Differentialgleichen zwischen $x, y$ die eine Gruppe von Transformationen gestatten. Math. Ann. 1888, 32, 213-281.

2. Arrigo, D.J. Symmetries Analysis of Differential Equations-An Introduction; Wiley: Hoboken, NJ, USA, 2015.

3. Bluman, G.; Kumei, S. Symmetries and Differential Equations; Springer: Berlin, Germany, 1989.

4. Cherniha, R.; Mykola, S.; Pliukhin, O. Nonlinear Reaction-Diffusion-Convection Equations: Lie and Conditional Symmetry, Exact Solutions and Their Applications; CRC Press: Boca Raton, FL, USA, 2018.

5. Olver, P.J. Applications of Lie Groups to Differential Equations, 2nd ed.; Springer: Berlin, Germany, 1993. 
6. Ovsiannikov, L.V. Gruppovye Svoystva Uravnenya Nelinaynoy Teploprovodnosty. Dok. Akad. Nauk. CCCP $1959,125,492$.

7. Ovsiannikov, L.V. Group Analysis of Differential Equations; Chapovsky, Y., Translator; Ames, W.F., Ed.; Academic Press: New York, NY, USA; London, UK, 1982.

8. Bluman, G.W.; Reid, G.J.; Kumei, S. New classes of symmetries for partial differential equations. J. Math. Phys. 1988, 29, 806-811.

9. Arrigo, D.J. Group Properties of a Monge-Ampere Equation. Ph.D. Thesis, Georgia Tech, Atlanta, GA, USA, 1991.

10. Ames, W.F.; Lohner, R.J.; Adams, E. Group properties of $u_{t t}=\left[f(u) u_{x}\right]_{x}$. Int. J. Non-Linear Mech. 1981, 16, 439-447.

11. Bluman, G.; Cheviakov, A.F.; Anco, S.C. Application of Symmetry Methods to Partial Differential Equations; Springer: Berlin, Germany, 2010.

12. Bluman, G.W.; Cheviakov, A.F. Framework for potential systems and nonlocal symmetries: Algorithmic approach. J. Math. Phys. 2005, 46, 123506.

13. Bluman, G.W.; Cheviakov, A.F.; Ivanova, N.M. Framework for nonlocally related partial differential equation systems and nonlocal symmetries: Extension, simplification, and examples. J. Math. Phys. 2006, 47, 113505.

14. Bluman, G.W.; Temuerchaolu; Sahadevan, R. Local and nonlocal symmetries for nonlinear telegraph equation. J. Math. Phys. 2005, 46, 023505.

15. Cheviakov, A.F.; Bluman, G.W. On locally and nonlocally related potential systems. J. Math. Phys. 2010, $51,073502$.

16. Yang, Z.; Cheviakov, A.F. Some realations between symmetries of nonlocally related systems. J. Math. Phys. 2014, 55, 083514.

17. Bluman, G.W.; Cheviakov, A.F. Nonlocally related systems, linearization and nonlocal symmetries for the nonlinear wave equation. Math. Anal. Appl. 2007, 333, 93-111.

18. Bluman, G.W.; Doran-Wu, P. The use of factors to discover potential systems or linearizations. Acta Appl. Math. 1995, 41, 21-43.

19. Bluman, G.; Kumei, S. On invariance properties of the wave equation. J. Math. Phys. 1987, 28, 307-319.

20. Akhatov, S.; Gazizov, R.K.; Ibragimov, N.K. Nonlocal symmetries. Heuristic approach. J. Sov. Math. 1991, 55, 1401-1450.

21. Sjöberg, A.; Mahomed, F.M. Non-local symmetries and conservation laws for one-dimensional gas dynamics equations. Appl. Math. Comput. 2004, 150, 379-397.

22. Bluman, G.; Cheviakov, A.; Ganghoffer, J.F. On the nonlocal symmetries, group invariant solutions and conservation laws of the equations of nonlinear dynamical compressible elasticitiy. In Proceedings of IUTAM Symposium on Progress in the Theory and Numerics of Configurational Mechanics, Erlangen, Germany, 20-24 October 2008; Steinmann, P., Ed.; Springer: Dordrecht, The Netherlands, 2009; pp. 107-120.

23. Arrigo, D.J.; Ashley, B.P.; Bloomberg, S.J.; Deatherage, T.W. Nonclassical Symmetries of a Nonlinear. Diffusion-Convection/Wave Equation and Equivalents Systems. Symmetry 2016, $8,140$.

24. Bruzon, M.S.; Gandarias, M.L.; Gonzalex, G.A.; Hansen, R. The $K(m, n)$ equation with a generalized evolution term studied by symmetry reductions and qualitative anslysis. Appl. Math. Comp. 2012, 218, 10094-10105.

25. Tychynin, V.A. Non-local symmetry and generating solutions for Harry-Dym type equations. J. Phys. A Math. Gen. 1994, 27, 4549-4556.

26. Bruzon, M.S.; Gandarias, M.L. Classical potential symmetries of the $K(m, n)$ equation with generalized evolution term. WSEAS Trans. Math. 2010, 4, 275-284.

27. Bluman, G.; Kumei, S. When nonlinear differential equations are equivalent to linear differential equations. SIAM J. Appl. Math. 1982, 42, 1157-1173.

28. Bluman, G.W.; Cole, J.D. The general similarity solution of the heat equation. J. Math. Phys. 1969, 18, $1025-1042$.

(C) 2018 by the authors. Licensee MDPI, Basel, Switzerland. This article is an open access article distributed under the terms and conditions of the Creative Commons Attribution (CC BY) license (http://creativecommons.org/licenses/by/4.0/). 